\title{
Comparison of the complete blood count-derived inflammatory markers in bipolar patients with manic and mixed episodes
}

\author{
Kirlioglu SS, Balcioglu YH, Kalelioglu T, Erten E, Karamustafalioglu N \\ Department of Psychiatry, Bakirkoy Prof Mazhar Osman Training and Research Hospital for Psychiatry, \\ Neurology, and Neurosurgery, Istanbul, Turkey. yhasanbalcioglu@gmail.com
}

\section{ABSTRACT}

OBJECTIVES: We aimed to compare altered inflammatory status between patients with bipolar manic and mixed episodes through neutrophil-to-lymphocyte ratio (NLR), platelet-to-lymphocyte ratio (PLR), and monocyte-tolymphocyte ratio (MLR) levels.

BACKGROUND: NLR, PLR, and MLR are systemic inflammation biomarkers that have recently studied in bipolar disorder (BD) manic and depressive episodes. Immunological biomarker signature of mixed episodes and MLR levels in BD have less been studied.

DESIGN AND SETTING: Our study included 48 bipolar patients in a mood episode (28 manic, 20 mixed) and 32 controls. Study-specific form including sociodemographic and clinical variables with laboratory findings were filled for all participants.

METHODS: Red cell distribution width (RDW), mean platelet volume (MPV) neutrophil, lymphocyte and platelet count, NLR, PLR, and MLR were recorded.

RESULTS: PLR and MLR were found significantly higher in bipolar patients compared to controls while NLR and MLR were significantly higher in manic patients than in mixed patients. RDW was found higher in mixed episode compared to controls.

CONCLUSIONS: One can interpret these findings as MLR would be considered as a novel state biomarker for bipolar mood episodes and greater inflammatory activation may be involved in mania rather than mixed episode (Tab. 2, Fig. 1, Ref. 35). Text in PDF www.elis.sk.

KEY WORDS: bipolar disorder, inflammation, mixed episodes.

\section{Introduction}

A particular attention has been increasingly devoted to understanding the role of immune and inflammatory mechanisms in the pathophysiology of bipolar disorder (BD). Immune system disturbances are considered as a cardinal determinant of neuroinflammatory processes that feature in both the etiology and course of BD (1); therefore, either in-vitro and clinical studies have indicated elevated peripheral immune-inflammatory signalling as a trait marker that represents neuroinflammation in the etiopathogenesis of $\operatorname{BD}(2,3)$. In fact, many studies have attempted to determine immune and inflammatory alterations in different mood episodes of BD, including elevated levels of tumor necrosis factor- $\alpha$ (TNF- $\alpha$ ), soluble TNF receptor type 1 (sTNF-R1), the soluble interleukin-2 receptor (sIL-2R) and activated lymphocyte cell subsets (4-9). However, the immune activation and elevated

Department of Psychiatry, Bakirkoy Prof Mazhar Osman Training and Research Hospital for Psychiatry, Neurology, and Neurosurgery, Istanbul, Turkey

Address for correspondence: Yasin Hasan Balcioglu, MD, Department of Psychiatry, Bakirkoy Prof Mazhar Osman Training and Research Hospital for Psychiatry, Neurology, and Neurosurgery, Postal code: 34147, Istanbul, Turkey.

Phone: +90212.4091515 inflammatory response could be associated across the variety of affective phases and BD patients exhibit a wide array of the level of inflammatory markers compared to healthy individuals $(3,10,11)$. Although the majority of the studies on inflammatory properties of BD have particularly focused on manic and depressive states, the immunological biomarker signature of mixed mood episodes has less been studied with revealing conflicting results (12). Neutrophil-to-lymphocyte ratio (NLR) and platelet-to-lymphocyte ratio (PLR) are simple, reliable, and cost-effective markers of systemic inflammatory response and easily measured by a complete blood count (CBC) $(13,14)$. NLR has been determined at high levels in patients with a poor prognosis of several malignancies, pancreatitis, and coronary heart disease $(15,16)$. NLR and PLR have been investigated in different psychiatric disorders such as manic and euthymic patients with BD and schizophrenia (17-19). Monocyteto-lymphocyte ratio (MLR) is another relatively new and simple marker that could be measured by CBC. MLR has been proposed as a surrogate marker for inflammation in certain patient populations, and it also has prognostic and predictive value in various disorders include haematologic, coronary disease and malignancy (20-22). A high monocyte count or a low lymphocyte count has been shown as an adverse effect of prognosis in these disorders. However, no studies have been conducted to utilize the relationship between MLR and neuropsychiatric disorders. 


\section{5-199}

In this study, we aimed to evaluate differences in inflammation via NLR, PLR, and MLR between BD patients with manic and mixed episodes. We also aimed to examine the possible relationship between the inflammation parameters and clinical manifestations of BD.

\section{Materials and methods}

\section{Study participants}

The present study included 80 participants, enrolled in-patients and healthy controls from January 2017 to January 2018. Forty-eight bipolar patients were recruited; 28 were in manic state, 20 were in mixed state, respectively. The patients' diagnoses confirmed by at least two senior psychiatrists based on Diagnostic and Statistical Manual of Mental Disorders, Fifth Edition (DSM-5) criteria within the first week of admission. All groups consisted of female individuals to obtain homogeneity of the data. Inclusion criteria for patients were; i) 18-65 years of age; ii) being hospitalized with the primary diagnosis of BD manic episode or BD manic or depressive episode with mixed features; iii) being drug-free at least one month before admission. Exclusion criteria were co-morbid psychiatric disorder, significant medical disorder (acute or chronic immuno-inflammatory disease) or pregnancy. A comparison group consisted of 32 healthy controls who were at the age between $18-65$, and blood donations are not accepted from patients with chronic disease or long-term drug use. None of the healthy control subjects were taking any form of prescribed or other medication and had no history of psychiatric disorders, dementia, or mental retardation in themselves or their first-degree relatives.

\section{Study procedure}

Data collection was provided from patients' files with a studyspecific form included socio-demographic and clinical characteristics. Following CBC components were also recorded; red cell distribution width (RDW), mean platelet volume (MPV) neutrophil, lymphocyte and platelet count, NLR, PLR, and MLR. Manic and depressive symptoms were assessed for the patient group using the Young Mania Rating Scale (YMRS) and the Hamilton Depression Rating Scale (HDRS) $(23,24)$. The validity and reliability of the Turkish versions of these scales were studied by Karadağ et al and Akdemir et al respectively $(23,25)$. This study was approved by the local ethical committee.

\section{Laboratory sampling}

Whole blood samples were obtained in the fasting from each subject between 8-10 a.m. using a standard venipuncture technique from antecubital veins. CBC samples were performed using a Sysmex XT1800i.

\section{Statistical analyses}

Data analyses were conducted using the statistical package for social sciences version 23.0 (SPSS). Kolmogorov-Smirnov test was used to examine whether the parameters are normally distributed. Kruskal-Wallis and Bonferroni corrected Mann-Whitney-U tests were used to analyze non-parametric data. ANOVA Bonferroni post-hoc statistical test was used for pairwise comparisons of account of MPV in multiple groups. Chi-square test was used to examine differences in categorical demographic and clinical variables. The relationship between the RDW, MPV, NLR, PLR and MLR levels and characteristic data of patients were analyzed

Tab. 1. Clinical and demographic characteristics of bipolar patients and healthy controls.

\begin{tabular}{|c|c|c|c|c|}
\hline & \multicolumn{2}{|c|}{ Bipolar participants $(n=48)$} & \multirow{2}{*}{$\begin{array}{l}\text { Healthy controls } \\
\qquad(\mathrm{n}=32)\end{array}$} & \multirow[b]{2}{*}{$\mathrm{p}$} \\
\hline & $\begin{array}{c}\text { Manic episode } \\
(\mathrm{n}=28)\end{array}$ & $\begin{array}{l}\text { Patients with mixed features } \\
\qquad(n=20)\end{array}$ & & \\
\hline $\mathrm{Age}^{1}($ years \pm SD) & $37.57 \pm 10.99$ & $36.55 \pm 8.33$ & $38.84 \pm 6.69$ & $0.784^{\mathrm{a}}$ \\
\hline Duration of illness ${ }^{2}$ (years \pm SD) & $11.32 \pm 8.94$ & $12.15 \pm 8.95$ & & $0.849^{\mathrm{b}}$ \\
\hline Age at onset ${ }^{1}($ years $\pm S D)$ & $26.17 \pm 7.59$ & $24.3 \pm 5.92$ & & $0.637^{\mathrm{b}}$ \\
\hline First treatment age ${ }^{1}$ & $26.71 \pm 7.69$ & $24.65 \pm 6.54$ & & $0.615^{\mathrm{b}}$ \\
\hline Number of hospitalizations ${ }^{2}$ & $5.32 \pm 6.8$ & $3.9 \pm 3.43$ & & $0.348^{\mathrm{b}}$ \\
\hline YMRS $^{1}$ & $32.53 \pm 9.34$ & $19.75 \pm 8.80$ & & $0.000^{\mathrm{b}, *}$ \\
\hline $\mathrm{HDRS}^{1}$ & & $36 \pm 8.37$ & & $0.187^{\mathrm{b}}$ \\
\hline
\end{tabular}

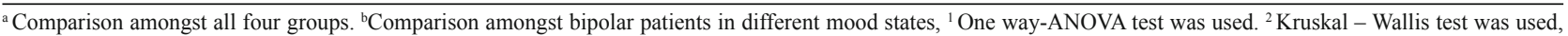
HDRS: Hamilton Depression Rating Scale. YMRS: Young Mania Rating Scale, " $p<0.05$ statistically significant, $\mathrm{SD}=$ standart deviation.

Tab. 2. Laboratory findings of groups.

\begin{tabular}{|c|c|c|c|c|c|c|c|c|}
\hline & $\begin{array}{l}\text { Manic episode }(\mathrm{n}=28) \\
\text { Mean } \pm \text { SD }\end{array}$ & $\begin{array}{l}\text { Patients with mixed features }(\mathrm{n}=20) \\
\qquad \text { Mean } \pm \text { SD }\end{array}$ & $\begin{array}{l}\text { Healthy controls }(n=32) \\
\text { Mean } \pm \text { SD }\end{array}$ & $p^{1}$ & $p^{2}$ & $p^{3}$ & $p^{4}$ & $p^{5}$ \\
\hline $\mathrm{NLR}^{\mathrm{a}}$ & $2.26 \pm 0.88$ & $1.93 \pm 1.18$ & $2.03 \pm 0.57$ & 0.077 & $0.040^{*}$ & 0.300 & 0.094 & 0.852 \\
\hline PLR $^{\mathrm{a}}$ & $103.9 \pm 20.7$ & $102.47 \pm 53.79$ & $116.35 \pm 35.15$ & $0.044^{*}$ & 0.099 & 0.192 & $0.023^{*}$ & $0.04^{*}$ \\
\hline $\operatorname{MLR}^{\mathrm{a}}$ & $0.31 \pm 0.1$ & $0.26 \pm 0.12$ & $0.22 \pm 0.06$ & $0.001^{*}$ & $0.038^{*}$ & $0.00^{*}$ & 0.463 & $0.004^{*}$ \\
\hline $\mathrm{RDW}^{\mathrm{a}}$ & $13.38 \pm 2.05$ & $13.70 \pm 1.02$ & $12.95 \pm 1.3$ & $0.042^{*}$ & 0.124 & 0.343 & $0.011^{*}$ & 0.051 \\
\hline $\mathrm{MPV}^{\mathrm{b}}$ & $9.40 \pm 1.67$ & $8.86 \pm 2.35$ & $8.96 \pm 2.02$ & 0.584 & 0.382 & 0.367 & 0.865 & 0.642 \\
\hline
\end{tabular}

$P^{1}$ : comparison amongst manic, patients with mixed features and control, $p^{2}$ :manic vs patients with mixed features, $p^{3}:$ manic vs control, $p^{4}$ : patients with mixed features vs

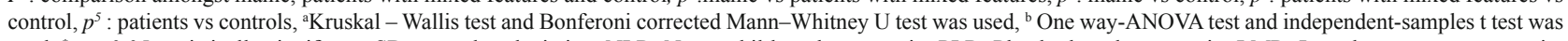
used, " $\mathrm{p}<0.05$ statistically significant, SD = standart deviation, NLR: Neutrophil lymphocyte ratio, PLR: Platelet lymphocyte ratio, LMR: Lymphocyte monocyte ratio, RDW: Red cell distribution width, MPV: Mean platelet volume 
through Spearman or Pearson Rank correlations tests. Results were assessed at significance $\mathrm{p}<0.05$ level.

\section{Results}

There was no significant difference between patients with manic episode, mixed state, and controls in terms of age (37.57 \pm 10.99 years, $36.55 \pm 8.33$ years and $38.84 \pm 6.69$ respectively, $\mathrm{p}>0.05$ ). Duration of illness, onset age of BD, age at first treatment, and the number of hospitalizations were not statistically significant between the groups. The demographic and clinical characteristics of BD patients and healthy subjects were summarized in Table 1.

NLR value of manic patients was $2.26 \pm 0.88$, patients with mixed features was $1.93 \pm 1.18$, and the control group was $2.03 \pm$ 0.57 . NLR value of manic patients was significantly higher than BD patients with mixed episode $(p<0.05)$. PLR value of mixed episode patients was significantly lower than in healthy controls $(\mathrm{p}$ $<0.05$ ), but there was no significant difference for PLR between manic and patients with mixed features. MLR value of the control group was significantly lower than in patients. In addition, MLR level of mixed episode patients was detected lower than manic patients. Kruskal-Wallis test results of NLR, PLR, and MLR values for three groups were demonstrated in Figure 1. BD patients with mixed features presented significantly higher RDW levels than controls, but the difference was not statistically significant between manic episode and mixed patients (Tab. 2). Correlation analysis did not reveal any significant finding between clinical variables and inflammatory variables.

\section{Discussion}

Our study is the first that reveal altered immune markers in mixed episode according to DSM-5 when compared with manic episode and healthy controls, despite relatively small sample size. Our main findings are that elevated levels of NLR and MLR in manic patients compared with mixed states. A recent study compared state specific, intraindividual alterations in levels of brainderived neurotrophic factor (BDNF), high-sensitivity C-reactive protein (hsCRP) and, cytokines in patients with BD during manic, depressive episodes, mixed states and compared with measurements in healthy control subjects and found that there were no differences between mixed states and manic states (26). However, in this study, it could be a potential confounder that all manic patients were medicated with high doses of antipsychotics and, as a consequence, it may reveal to decrease levels of inflammatory markers $(27,28)$. Although, we included patients were drug-free at least one month before admission to prevent possible effects of antipsychotic drugs to the immune-inflammatory system.

There is a suggestion in a previous research demonstrating increased levels of pro-inflammatory cytokines IL- 6 and TNF- $\alpha$ during an acute manic episode of bipolar disorder determined that the increased activity of pro-inflammatory cytokines and an imbalance between pro-inflammatory and anti-inflammatory cytokines may play a role in the pathophysiology of episodic process in bipolar
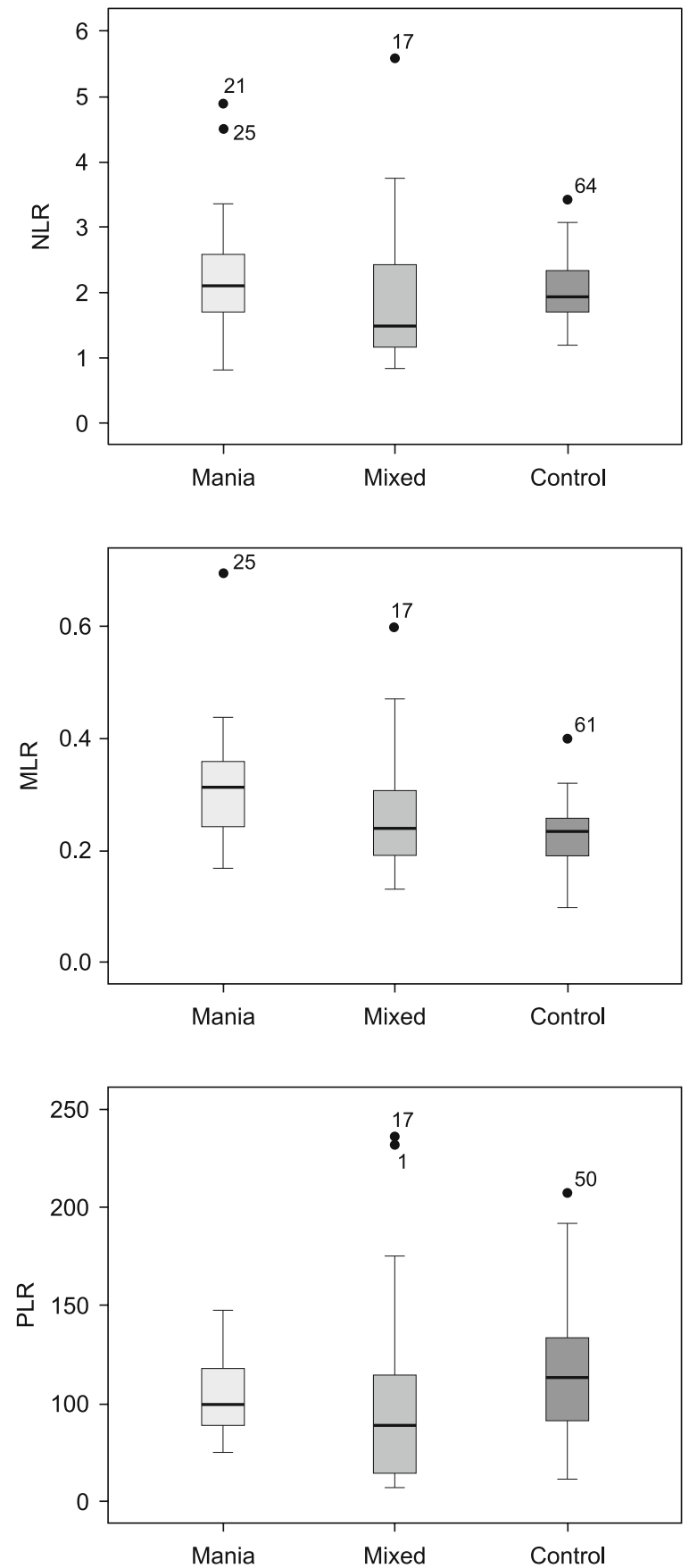

Fig. 1. Independent-samples Kruskal-Wallis test for NLR, MLR, and PLR in mania, mixed, and control groups.

disorder (1). Rajkowska et al hypothesized that activated microglia in the brain activated the release of pro-inflammatory cytokines which mediates negative effects on the neuroprotective system, thereby exacerbating pathophysiological disturbances in BD (29). Another theory suggests that inflammatory substances reveal to destabilization of the brain function, effected with environmental factors and, accordingly contribute to the episodic presentation 
of mood symptoms in $\mathrm{BD}(30,31)$. Both lithium and valproate (VPA) in BD may be partly attributable via their anti-inflammatory properties. Lithium and VPA reveal microglial inactivation and reduces overexpression of proinflammatory cytokines, including nitric oxide synthase, and COX-2 (32). Consequently, the moodstabilizing effect of these drugs requires a regulation in inflammatory intracellular cascades involves proinflammatory eicosanoids, cytokines, and acute-phase proteins, which are elevated during acute mood episodes (33).

In this present study, we have determined that potential peripheral inflammation markers elevated levels of NLR and MLR during manic episode in bipolar patients when compared with mixed states. In a recent study, Bai et al reported that the patients in a depressive state had significantly lower levels of sTNF-R1 than the patients in manic/hypomanic and euthymic states (34). Similarly, another study comparing the difference in hsCRP levels among bipolar patients with different mood states demonstrated that serum levels of hsCRP were increased in bipolar patients during manic episode, as compared to euthymic, depressed patients and healthy controls (35). We considered that depressive components of mixed states may reveal a low-grade inflammation by comparison with manic episode in the light of above-mentioned studies and our findings.

Although NLR and PLR have been studied in bipolar disorder before, to the best of our knowledge, this is the first study regarding the levels of MLR in patients with bipolar disorder, particularly comparison between manic and mixed states $(17,18)$. We have demonstrated significantly higher MLR values in the manic episode compared to mixed states, while increased MLR levels were determined in the bipolar disorder patients compared to the control subjects. Increments of MLR values is a novel finding. Thus, there may be higher inflammatory response during the manic episode than mixed and depressive states in bipolar disorder.

Limitations of the current study were its cross-sectional nature and relatively small sample size. Although including only female gender may not be representative of bipolar population, we eliminated the gender differences on $\mathrm{CBC}$ which may be both advantage and limitation of our study. Another methodological difficulty for patients was that; even though the patients were medication free for at least one month, we couldn't rule out the individuals who received PRN (pro re nata) medication at admission.

\section{Conclusion}

In conclusion, we found that increased levels of NLR and MLR in BD patients during manic episodes, as compared with mixed states. According to this findings, differences in these potential peripheral inflammation markers between manic and mixed featured episodes may result from the depressive components of mixed states. In addition, the present study is the first to investigate MLR in patients with BD and this marker supports the higher levels of inflammatory activation in manic episodes. Longitudinal studies including depressive states are necessary to better determine the clinical consequences of activation of inflammatory systems during episodes in BD.

\section{Highlights}

- NLR and PLR have been previously found in relation with bipolar manic episode while MLR have not been studied in mood episodes yet.

- The immunological biomarker signature of mixed mood episodes has less been studied with revealing conflicting results.

- PLR and MLR were found significantly higher in bipolar patients compared to controls; MLR would be considered as a novel inflammatory biomarker for bipolar mood episodes.

- NLR and MLR were significantly higher in manic patients than in mixed patients; greater inflammatory activation may be involved in mania rather than mixed episode.

\section{References}

1. Kim YK, Jung HG, Myint AM, Kim H, Park SH. Imbalance between pro-inflammatory and anti-inflammatory cytokines in bipolar disorder. J Affect Disord 2007; 104 (1-3): 91-95.

2. Goldstein BI, Kemp DE, Soczynska JK, McIntyre RS. Inflammation and the phenomenology, pathophysiology, comorbidity, and treatment of bipolar disorder: a systematic review of the literature. J Clin Psychiatry 2009; 70 (8): 1078-1090.

3. Berk M, Kapczinski F, Andreazza AC, Dean OM, Giorlando F, Maes $\mathbf{M}$ et al. Pathways underlying neuroprogression in bipolar disorder: Focus on inflammation, oxidative stress and neurotrophic factors. Neurosci Biobehav Rev 2011; 35: 804-817.

4. Tsai SYM, Yang YY, Kuo CJ, Chen CC, Leu SJC. Effects of symptomatic severity on elevation of plasma soluble interleukin-2 receptor in bipolar mania. J Affect Disord 2001; 64 (2-3): 185-193.

5. Barbosa IG, Huguet RB, Mendonça VA, Sousa LP, Neves FS, Bauer ME et al. Increased plasma levels of soluble TNF receptor I in patients with bipolar disorder. Eur Arch Psychiatry Clin Neurosci 2011; 261 (2): 139-143.

6. Tsai SY, Chen KP, Yang YY, Chen CC, Lee JC, Singh VK et al. Activation of indices of cell-mediated immunity in bipolar mania. Biol Psychiatry 1999; 45 (8): 989-994.

7. Munkholm K, Vinberg M, Berk M, Kessing LV. State-related alterations of gene expression in bipolar disorder : a systematic review. Bipolar Disord 2012; 684-696.

8. Breunis MN, Kupka RW, Nolen WA, Suppes T, Denicoff KD, Leverich GS et al. High numbers of circulating activated T cells and raised levels of serum IL-2 receptor in bipolar disorder. Biol Psychiatry 2003; 53 (2): $157-165$.

9. Drexhage RC, Hoogenboezem TH, Versnel MA, Berghout A, Nolen WA, Drexhage HA. Brain, Behavior, and Immunity The activation of monocyte and $\mathrm{T}$ cell networks in patients with bipolar disorder. Brain Behav Immun 2011; 25 (6): 1206-1213.

10. Rosenblat JD. Bipolar Disorder and Inflammation. Psychiatr Clin NA 2016; 39 (1): 125-137.

11. O'Brien SM, Scully P, Scott L V, Dinan TG. Cytokine profiles in bipolar affective disorder: focus on acutely ill patients. J Affect Disord 2006; 90 (2-3): 263-267.

12. Haenisch F, Alsaif M, Guest PC, Rahmoune H, Yolken RH, Dickerson F et al. Multiplex immunoassay analysis of plasma shows differences in biomarkers related to manic or mixed mood states in bipolar disorder patients. J Affect Disord 2015; 185: 12-16. 
13. Zahorec R. Ratio of neutrophil to lymphocyte counts - rapid and simple parameter of systemic inflammation and stress in critically ill. Bratisl Lek Listy 2001; 102 (1): 5-14.

14. Balta S, Aparcı M, Ozturk C, Demirkol S, Celik T. Neutrophillymphocyte ratio as an useful mortality marker. Am J Emerg Med 2014; 32 (12): 1546-1547.

15. Turkmen K, Guney I, Yerlikaya FH, Tonbul HZ. The Relationship Between Neutrophil-to-Lymphocyte Ratio and Inflammation in End-Stage Renal Disease Patients. Ren Fail 2012; 34 (2): 155-159.

16. Semple JW, Freedman J. Platelets and innate immunity. Cell Mol Life Sci 2010; 67 (4): 499-511.

17. Kalelioglu T, Akkus M, Karamustafalioglu N, Genc A, Genc ES, Cansiz A et al. Neutrophil-lymphocyte and platelet-lymphocyte ratios as inflammation markers for bipolar disorder. Psychiatry Res 2015; 228 (3): 925-927.

18. Özdin S, Sarisoy G, Böke Ö. A comparison of the neutrophil-lymphocyte, platelet-lymphocyte and monocyte-lymphocyte ratios in schizophrenia and bipolar disorder patients - a retrospective file review. Nord J Psychiatry 2017; 71 (7): 509-512.

19. Semiz M, Yildirim O, Canan F, Demir S, Hasbek E, Tuman TC et al. Elevated neutrophil/lymphocyte ratio in patients with schizophrenia. Psychiatr Danub 2014; 26 (3): 220-225.

20. Chen L, Zhang F, Sheng XG, Zhang SQ. Decreased pretreatment lymphocyte/monocyte ratio is associated with poor prognosis in stage Ib1IIa cervical cancer patients who undergo radical surgery. Onco Targets Ther 2015; 8: 1355-1362.

21. Belotti A, Doni E, Bolis S, Rossini F, Casaroli I, Pezzatti S et al. Peripheral blood lymphocyte/monocyte ratio predicts outcome in follicular lymphoma and in diffuse large B-cell lymphoma patients in the rituximab era. Clin Lymphoma Myeloma Leuk 2015; 15 (4): 208-213.

22. Szkandera J, Gerger A, Liegl-Atzwanger B, Absenger G, Stotz $\mathbf{M}$, Friesenbichler $\mathbf{J}$ et al. The lymphocyte/monocyte ratio predicts poor clinical outcome and improves the predictive accuracy in patients with soft tissue sarcomas. Int J Cancer 2014; 135 (2): 362-370.

23. Karadağ F, Oral T, Yalçin FA, Erten E. Reliability and validity of Turkish translation of Young Mania Rating Scale. Turk Psikiyatri Derg 2002; 13 (2): 107-114.
24. Hamilton M. A rating scale for depression. J Neurol Neurosurg Psychiatry 1960; 23 (1): 56-62.

25. Akdemir A, Türkçapar MH, Orsel SD, Demirergi N, Dag I, Ozbay MH. Reliability and validity of the Turkish version of the Hamilton Depression Rating Scale. Compr Psychiatry 2001; 42 (2): 161-165.

26. Jacoby AS, Munkholm K, Vinberg M, Pedersen BK, Kessing LV. Cytokines, brain-derived neurotrophic factor and C-reactive protein in bipolar I disorder - Results from a prospective study. J Affect Disord 2016; 197: 167-174.

27. Fernandes BS, Steiner J, Bernstein H-G, Dodd S, Pasco JA, Dean OM et al. C-reactive protein is increased in schizophrenia but is not altered by antipsychotics: meta-analysis and implications. Mol Psychiatry 2016; 21 (4): 554-564.

28. Baumeister D, Ciufolini S, Mondelli V. Effects of psychotropic drugs on inflammation: consequence or mediator of therapeutic effects in psychiatric treatment? Psychopharmacology (Berl) 2016; 233 (9): 1575-1589.

29. Rajkowska G. Postmortem studies in mood disorders indicate altered numbers of neurons and glial cells. Biol Psychiatry 2000; 48 (8): 766-777.

30. Leonard BE. The immune system, depression and the action of antidepressants. Prog Neuro-Psychopharmacology Biol Psychiatry 2001; 25 (4): 767-780.

31. Smith RS, Maes M. The macrophage-T-lymphocyte theory of schizophrenia: additional evidence. Med Hypotheses 1995; 45 (2): 135-141.

32. Chiu CT, Wang Z, Hunsberger JG, Chuang DM. Therapeutic potential of mood stabilizers lithium and valproic acid: beyond bipolar disorder. Pharmacol Rev 2013; 65 (1): 105-42

33. Grunze H. Bipolar Disorder.In: Zigmon MJ, Coyle JT, Rowland LP, editors. Neurobiology of Brain Disorders. Academic Press; 2015. p. 655-673.

34. Bai YM, Su TP, Tsai SJ, Wen-Fei C, Li CT, Pei-Chi T et al. Comparison of inflammatory cytokine levels among type I/type II and manic/ hypomanic/euthymic/depressive states of bipolar disorder. J Affect Disord 2014; 166: 187-192.

35. Cunha AB, Andreazza AC, Gomes FA, Frey BN, Da Silveira LE, Gonçalves CA et al. Investigation of serum high-sensitive C-reactive protein levels across all mood states in bipolar disorder. Eur Arch Psychiatry Clin Neurosci 2008; 258 (5): 300-304. 